\title{
Enriching a Situation Awareness Framework for IoT with Knowledge Base and Reasoning Components
}

\author{
Niklas Kolbe ${ }^{1}$, Arkady Zaslavsky ${ }^{2,3}$, Sylvain Kubler ${ }^{1}$, Jérémy Robert ${ }^{1}$, and Yves \\ Le Traon ${ }^{1}$ \\ 1 University of Luxembourg, Interdisciplinary Center for Security, Reliability and \\ Trust, 4 rue Alphonse Weicker, L-2721 Luxembourg, Luxembourg \\ \{niklas.kolbe, sylvain.kubler, jeremy.robert, yves.letraon\}@uni.lu \\ 2 Commonwealth Scientific and Industrial Research Organisation, Data61, Clayton \\ VIC 3168, Australia \\ arkady.zaslavsky@csiro.au \\ 3 Saint Petersburg National Research University of ITMO, 49 Kronverksky Pr., St. \\ Petersburg, 197101, Russia \\ arkady.zaslavsky@acm.org
}

\begin{abstract}
The importance of system-level context- and situation awareness increases with the growth of the Internet of Things (IoT). This paper proposes an integrated approach to situation awareness by providing a semantically rich situation model together with reliable situation inference based on Context Spaces Theory (CST) and Situation Theory (ST). The paper discusses benefits of integrating the proposed situation awareness framework with knowledge base and efficient reasoning techniques taking into account uncertainty and incomplete knowledge about situations. The paper discusses advantages and impact of proposed context adaptation in dynamic IoT environments. Practical issues of two-way mapping between IoT messaging standards and CST are also discussed.
\end{abstract}

Keywords: Context Space Theory, Situation Awareness, Situation Theory, Ontology, O-MI/O-DF, Context Adaptation

\section{Introduction}

With the growth of the Internet of Things (IoT) more and more devices publish sensed information, promoting the development of smart services and pervasive computing systems. The key feature of such systems is called context awareness; i.e. computing systems become aware of their state and environment in which they run, and provide services of higher value to humans [1]. Existing implementations of pervasive computing systems include for example home automation, smart energy systems, decision support systems for emergency cases, environmental impact monitoring, improved efficiency of transportation systems and many more. Furthermore, the IoT is considered as a key technology that could potentially enable the transition to a more sustainable society by providing the necessary 
information for a fundamental change in the way societies produce goods, use products, and consume resources as well as services. The advances towards sustainability are driven by large-scale problems and global challenges like resource depletion, food security, and climate change, as for instance stated by the Ellen MacArthur Foundation ${ }^{4}$. Still, several research challenges in various disciplines remain unsolved to deploy IoT-based systems more vastly and in a bigger scope, particularly regarding its pervasive feature.

A pervasive computing system needs to interpret acquired sensor data to fully understand the environment. Whereas context refers to features of entities [1], situations are of a more complex structure, e.g. involving relations between entities, temporal aspects, and requiring additional semantic interpretation. Situation aware applications thus rely on the integration of external knowledge to achieve an understanding of the environment on a higher level of abstraction than context [23]. This paper is concerned with challenges in knowledge representation and reasoning to achieve situation awareness in a general way, and discusses several issues that need to be considered when developing the knowledge base. The paper is built upon prior work: A domain-independent approach combining various concepts, e.g. ontologies, Context Space Theory (CST), Situation Theory and IoT messaging standards to provide a holistic framework for situation awareness [14]. Based on this framework, the following discussions cover the knowledge base integration, issues that are caused by incomplete knowledge about situations, and the implications of adaptation to current context for the knowledge base.

Section 2 presents related work and background in the domain of ontologies as knowledge base for situation aware systems. Section 3 introduces the framework that is considered in this paper to achieve situation awareness. It forms the foundation for discussions in the subsequent sections. Section 4 is concerned with defining and handling the knowledge base and preprocessing it for runtime reasoning. Sections 5 and 6 deal with incomplete knowledge and context adaptation related to the knowledge base respectively; the conclusion follows.

\section{Ontology-based Situation Awareness}

Ontologies are defined as an "explicit specification of a conceptualization" [12] and have often been applied to develop a general model for situation awareness. The advantages of ontologies as a technique for situation modelling over other techniques - like graph models, object-role modeling, markup schemes, and spatial logic - are the eligibility of handling the heterogeneity of sensor data, capturing relationships and dependencies between context information and the native support for reasoning [3]. Moreover, "being understandable, shareable, and reusable by both humans and machines" [23] is another important characteristic of ontologies, because it supports the complex and error-prone process of gathering and maintaining required domain knowledge. Ontologies can also be integrated into the existing infrastructures of relevant computing environments [20].

\footnotetext{
${ }^{4}$ Ellen MacArthur Foundation, Report on Intelligent Assets: https://www.ellenmacarthurfoundation.org/publications/intelligent-assets
} 
The presented features of ontologies reduce the knowledge engineering efforts, which motivates the incorporation of ontologies into a general situation awareness framework. However, ontologies have known limitations in the native reasoning support and performance issues during run-time in large-scale systems. Thus, the consideration of ontologies is restricted to the modelling and initialization phase of the system.

\subsection{Situation Models and Upper Ontologies}

Semantic web technologies like RDF, RDFS, OWL, and ontology reasoners, provide tools to share and reuse defined knowledge and are thus a feasible technology to develop a knowledge base for situation aware systems in IoT settings. The design of ontologies for this purpose needs to comply with semantic requirements regarding the capabilities of representing contexts and situations in a general way. In the following, a brief overview of existing approaches to define such situation models in the form of domain-independent, so-called upper ontologies, is presented.

Upper ontologies that were developed to provide a context or situation model have already been surveyed, for example in [2]. The authors developed an evaluation framework with context- and situation-related criteria and compared the design of four ontologies, namely SAWA [15], Situation Ontology [22], SOUPA [7] and CONON [21]. It is concluded that ontologies which are primarily targeted for context awareness (SOUPA and CONON) do not comply with the criteria for a general situation model. Most of the defined criteria are met by the SAWA ontology, however, even this approach lacks the support for situation types, roles and representation of space and time.

Other related approaches include the Situational Context Ontology [3]. The model combines contextual information (spatial and temporal) with related situations of an individual. Situation definitions are not entities of the ontology definition itself, but formulated externally with rules based on the Semantic Web Rule Language (SWRL).

The Situation Theory Ontology [13] is based on the semantics of the Situation Theory [10]. In Situation Theory, facts about the world are denoted as infons. An infon is defined as a relation among objects (e.g. individuals, attributes or other situations) with a polarity that defines whether this relation is true or false. A situation is characterized by the set of infons that this situation supports. Moreover, the Situation Theory supports logical operators and parameters representing types of objects and situations for more complex statements which can also be formulated in the Situation Theory Ontology.

\subsection{Requirements for Situation Models to support Reasoning}

Further considerations for the situation model have to be taken into account to allow for reliable reasoning results and practical applicability of the approach. These include for example mobility, timeliness as well as uncertainty (imperfection and ambiguity) [3] in the model. Furthermore, incompleteness of knowledge, 
distributed composition of the system [20] - e.g. incorporation of sensor data from various sources and interoperability aspects - and the ability of the system to adapt current context may have implications on the requirements of the situation model. In this paper, the discussion is limited to aspects of uncertainty, incompleteness, sensor data integration, and dynamic context adaptation, related to ontology-based situation models.

Uncertainty aspects of a pervasive computing system, i.e. imperfect sensor data (e.g. missing values, imprecision) and incomplete knowledge about situations, are often approached with fuzzy logic. In fuzzy logic, membership functions can be defined, which map a set of numerical values to a fuzzy shape. The rule-based reasoning calculates a membership degree (between 0 and 1) for each fuzzy set, since the conditions for the sets may overlap. One approach that combines fuzzy logic and ontologies for situation awareness is the Fuzzy Situation Theory Ontology (FSTO) [11]. It extends the polarity of the infons of Situation Theory. Instead of assigning boolean values to the relation among objects, vagueness can be added through expressions like quite true. Situation occurrence is inferred through a model which considers the membership functions for infons and situations.

From a holistic viewpoint of a situation aware approach, the integration of sensor data also plays an important role; both from a modelling perspective (i.e., linking sensor data from physical data to modelled context) as well as an interoperability perspective (i.e., acquiring sensor data in an IoT setting). An example and W3C standard to model a sensor setup in an ontology is the Semantic Sensor Network (SSN) ontology [8]. SSN defines the relation between the network setup, sensing devices, and their observations (i.e. sensor data readings). In an effort to provide a similar model for actuators, the SAN ontology ${ }^{5}$ was developed. Both ontologies were applied for example in [19] to provide a complete IoT ontology.

Smart, situation aware services in the IoT are based on the access to contextual information and other services that often reside in domain-specific platforms (also referred to as vertical silos in the IoT), where interoperability for cross-domain and -platform services is difficult to achieve. In the framework of this research, the IoT messaging standards of the bIoTope $\mathrm{H} 2020$ project $^{6}$ are considered to achieve cross-platform interoperability, namely the O-MI (Open-Messaging Interface $^{7}$ ) and O-DF (Open-Data Format ${ }^{8}$ ) standards. These provide a generic and standardized way to expose and describe contextual data on top of the techniques used by different platforms, which makes the access to contextual data and thus the situation aware component domain-independent.

\footnotetext{
${ }^{5}$ SAN ontology: https://www.irit.fr/recherches/MELODI/ontologies/SAN.owl

${ }^{6}$ bIoTope project: http://www.biotope-project.eu/

7 Open-Messaging Interface: https://www2 .opengroup.org/ogsys/catalog/C14B

8 Open-Data Format: https://www2.opengroup.org/ogsys/catalog/C14A
} 


\section{Framework based on Context Space Theory}

This section introduces the framework on which the work of this paper is based on (originally presented in [14]). The combination of different techniques is necessary to meet the aforementioned requirements for a situation aware system, as the exclusive use of ontologies is not sufficient to provide reliable reasoning. The presented approach is based on ontologies for modelling and merged with the Context Space Theory for reasoning and O-MI/O-DF for sensor data acquisition.

The Context Space Theory [17] was developed for reasoning about context based on a multidimensional spatial metaphor. Fig. 1 visualizes the main concepts of this theory. Context attributes, which are measurable properties usually provided by sensors, form the dimensions of the context or application space. Real-life situations are represented as subspaces (illustrated as multi-dimensional bubbles) of the application space, named situation spaces. The context state describes a point that moves through the application space depending on the current values of a corresponding set of context attribute values over time (as represented by the dashed line). If the context state lies in the subspace of a situation, this situation is occurring. This inference is based on a place-holder function that allows the usage of different techniques to reason about situation occurrence.

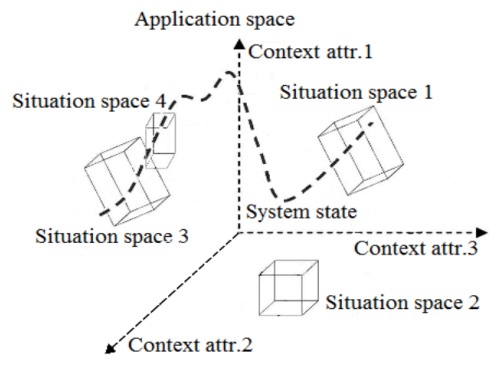

Fig. 1. Illustration of the Context Space Theory [6]

The overall architecture of the framework and underlying building blocks (based on ECSTRA [4]) are displayed in Fig. 2. The upper ontology designed for this framework is based on STO and SSN. The concepts of these different ontologies were mapped and specific CST-related aspects were added to the ontology (presented in [14]). The upper ontology is used to capture domain- and application-specific knowledge about situations, individuals, attributes, sensors, actuators, and their relations. The combined usage of these upper ontologies provides a complete specification of all relevant knowledge assets relevant for a situation aware system.

The information provided by this knowledge base is used for the initialization of the system, i.e. to generate the situation spaces in the context space based on the situation definitions in the ontology. Furthermore, the situation definitions 


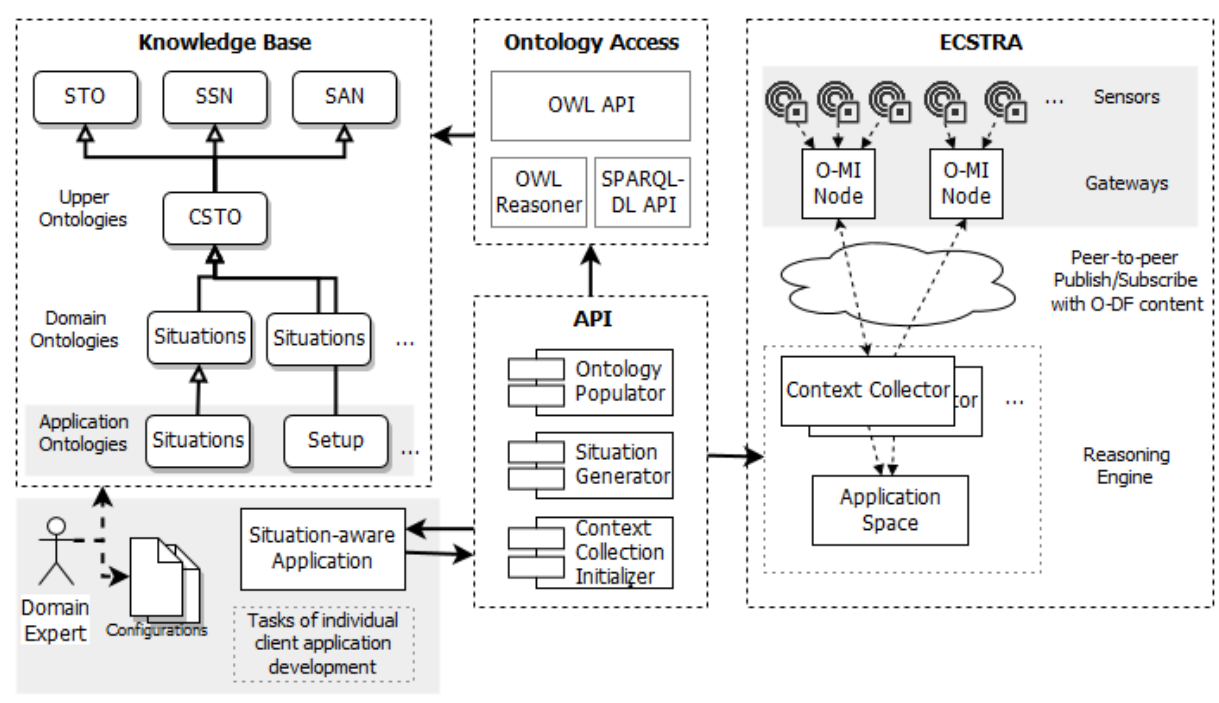

Fig. 2. Framework Architecture for Situation Awareness

from the ontology can be used to assign sensor data to relevant context attributes and involved individuals in situations, which extends the concepts defined in CST.

A related approach that combined CST and ontologies is presented in paper [5]. In this approach, a context ontology (CONON) and a sensor ontology (SSN) are used as a foundation to develop situation formulas which are then translated to Context Space.

\section{Integration of the Ontological Knowledge Base into the Reasoning System}

This section presents the incorporation of STO as a knowledge base for a CSTbased reasoning approach. Firstly, the situation representations of STO and CST are mapped, in order to extract the knowledge from the ontology and to create the situation spaces in the context space. Secondly, issues are discussed that occur when the situation definitions are translated to the CST model, and how additional processing allows for situation space generation with reduced knowledge engineering effort.

\subsection{Mapping of Situation Representations in ST and CST}

In the following, both the formal representation of situations in CST and in ST are presented in order to develop a formal mapping of these concepts. Both theories and their formal denotations are summarized in Table 1, moreover, it also 
shows the mapping of the individual representations by assigning corresponding, general concepts.

In CST, a situation space $S_{j}$ is formally defined by an acceptable region $A_{a_{i}}^{S_{j}}$ for each corresponding context attribute $a_{i}$, which form the dimensions of this situation space in the overall context space. An acceptable region $A_{a_{i}}^{S_{j}}$ contains the elements that satisfy a predicate $P(V)$ and thus, defines the shape of the multidimensional bubble.

Inferring whether a situation is occurring is based on the context state, which is the set of current values of all context attributes. The inference function $\mu_{S_{j}}$ uses a Dempster-Shafer approach to calculate a confidence level for situation is occurrence. For this, the inference function takes the general relevance and the contribution of the current value of each context attribute into account. The relevance function $w_{S_{j}}\left(a_{i}\right)$ formally assigns a weight $w \in[0,1]$ to each context attribute $a_{i}$ of a situation space $S_{j}$. This weight defines the relative importance of a particular context attribute to infer a situation occurrence. To allow more refined specifications, the contribution function $\eta_{a_{i}}^{S_{j}}\left(x_{a_{i}}\right)$ assigns a contribution degree $c$ for each value $x_{a_{i}}$ in an acceptable region for a situation space. If, for example, the value of a context attribute is at the border of a situation space, it might be uncertain whether this value contributes to the occurrence of the situation, thus, this value within the acceptable region can be assigned with a lower contribution degree. The calculated confidence value for situation occurrence by $\mu_{S_{j}}$ is compared to a confidence threshold $\varepsilon_{i}$ to get a boolean output.

In Situation Theory [10], on the other hand, situations are defined by specifying which infons they support, denoted as $s \models \sigma$. Infons represent facts about the world and are defined as a relation $r$ among $n$ objects, with the polarity $i$ whether this relation is true or false: $\sigma=\ll r, a_{1}, \ldots, a_{n}, i \gg$. Objects that stand in a relation can be individuals, attributes and situations. Moreover, ST considers the definition of types and parameters to make statements about a group of objects, which were extended for STO. ATTR, SIT and VAL for example represent the types of attributes, situations and values correspondingly.

As already mentioned, Table 1 not only summarizes these definitions presented before but also shows how these concepts of the two theories correspond to each other. Consequently, it can be inferred that the basic composition of a situation space can be extracted from the situation definition in ST. Information assets that are required for CST-based reasoning, however, cannot be extracted from native ST definitions. Thus, the situation specification in STO requires extensions to allow the integration of all required assets in the knowledge base. On the other hand, ST comes with concepts that are not considered in CST that can enhance the reasoning capabilities, as presented in the following subsection.

\subsection{On Involved Individuals and Type Definitions}

Situation reasoning is not only dependent on the context attributes, but also on individuals - items, persons, objects, etc. - that are involved in the situation. In 
Table 1. Mapping of CST and ST Concepts for Situation Representation

\begin{tabular}{|c|c|c|}
\hline Concept & CST Representation & ST Representation \\
\hline Situation & $\begin{array}{l}\text { Situation Space } \\
S_{j}\end{array}$ & $\begin{array}{l}\text { Situation } \\
s\end{array}$ \\
\hline Attribute & $\begin{array}{l}\text { Context Attribute } \\
a_{i}\end{array}$ & $\begin{array}{l}\text { Attribute } \\
a_{n} \text { type } A T T R\end{array}$ \\
\hline $\begin{array}{l}\text { Values \& } \\
\text { Ranges }\end{array}$ & $\begin{array}{l}\text { Acceptable Region } \\
A_{a_{i}}^{S_{j}}=\{V \mid P(V)\}\end{array}$ & $\begin{array}{l}\text { Values } \\
a_{n} \text { type } V A L \text { in } \sigma\end{array}$ \\
\hline $\begin{array}{l}\text { Situation } \\
\text { Composition }\end{array}$ & $\begin{array}{l}\text { Set of Acceptable Regions } \\
S_{j}=\left(A_{1}^{j}, A_{2}^{j}, \ldots, A_{n}^{j}\right)\end{array}$ & $\begin{array}{l}\text { Set of Supported Infons } \\
s \models \sigma \\
\sigma=\ll r, a_{1}, \ldots, a_{n}, i \gg\end{array}$ \\
\hline State & $\begin{array}{l}\text { Context State } \\
x=\left(x_{a_{1}}, x_{a_{2}}, \ldots, x_{a_{n}}\right) \\
x_{a_{i}}: \text { value of } a_{i}\end{array}$ & - \\
\hline $\begin{array}{l}\text { Attribute } \\
\text { Weight }\end{array}$ & $\begin{array}{l}\text { Relevance } \\
w_{S_{j}}\left(a_{i}\right)=w \\
w \in[0,1] ; \sum_{i=1}^{n} w_{S_{j}}\left(a_{i}\right)=1\end{array}$ & - \\
\hline State Weight & $\begin{array}{l}\text { Contribution } \\
\eta_{a_{i}}^{S_{j}}\left(x_{a_{i}}\right)=c ; c \in[0,1]\end{array}$ & - \\
\hline Inference & $\begin{array}{l}\text { Inference Function } \\
\mu_{S_{j}}=\sum_{i=1}^{n} w_{S_{j}}\left(a_{i}\right) * \eta_{a_{i}}^{S}\left(x_{a_{i}}\right) \\
\text { Confidence Threshold } \varepsilon_{i}\end{array}$ & - \\
\hline $\begin{array}{l}\text { Type } \\
\text { Abstraction }\end{array}$ & - & $\begin{array}{l}\text { Situation Types, Object Types } \\
{[\dot{s} \mid \dot{s} \models \sigma], \dot{a_{n}}}\end{array}$ \\
\hline $\begin{array}{l}\text { Involved } \\
\text { Individuals }\end{array}$ & - & $\begin{array}{l}\text { Involved Individuals } \\
a_{n} \text { type } I N D\end{array}$ \\
\hline
\end{tabular}

CST, multiple involved individuals for one situation definition can be handled in two different ways. The first approach is to maintain multiple context states simultaneously and to reason about a general situation space, whereas in the second approach a single context state is maintained and reasoning is performed over dedicated situation spaces for each involved individual. For example, the second case needs to be considered if situation spaces of a type definition differ depending on the involved individual, and if the situation space moves over time depending on reasoning results. As discussed before, the context values from external sources are linked to attributes, individuals, and situation definitions by extending the model with the SSN ontology.

The support of type abstraction is an important feature to keep the complexity of the modelling process low. ST facilitates such general specifications through 
the use of (abstraction) parameters in situation type definitions. As defined in [10], situation types are formally denoted as $[\dot{s} \mid \dot{s} \models \sigma]$. The transformation from a situation definition to a situation space depends on whether the situation definition is based on objects or types, as explained in the following.

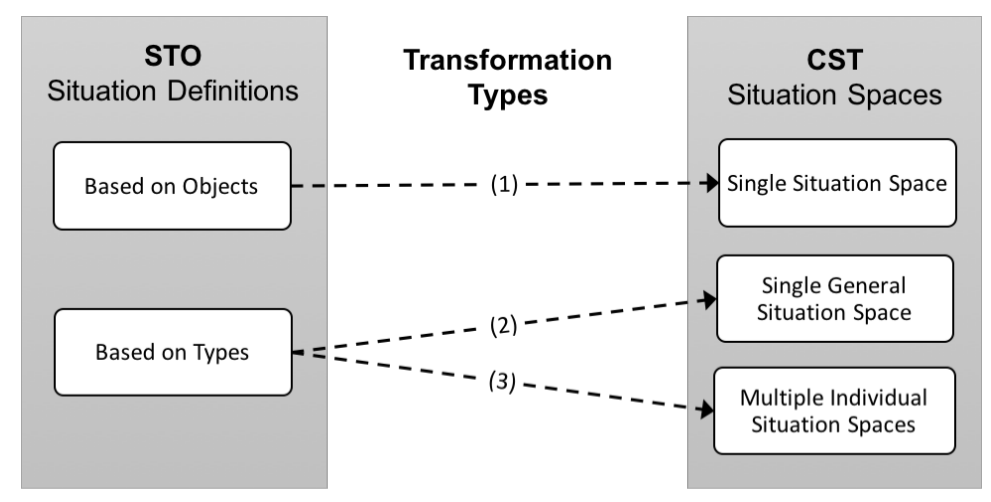

Fig. 3. Transformation from Situation Definition to Situation Space

The situation space generation has to be handled in different ways based on the situation definition in ontology. Fig. 3 shows a classification of the transformation types from situation representation in ST to CST.

If a situation definition is solely based on objects it can be transformed to a corresponding single situation space in a straight-forward manner. The transformation is formally denoted in Eq. (1).

$$
s \models \sigma \Rightarrow S_{j}
$$

If a situation definition in STO includes type abstraction two approaches for situation generation can be differentiated, based on the considerations in the beginning of this section. The first option, denoted in Eq. (2), represents the generation of a general situation space for a set of involved individuals.

$$
[\dot{s} \mid \dot{s} \models \sigma] \Rightarrow S_{j}
$$

The second option for a type-based transformation, formally given in Eq. (3), generates multiple situation spaces. In the first step, all possible situations based on objects are derived from the type definition by calculating the $n$-fold Cartesian product over the objects of the $n$ type definitions involved in the situation definition. In the second step, the resulting definitions based on objects are transformed to corresponding situation spaces.

$$
[\dot{s} \mid \dot{s} \models \sigma] \Rightarrow\{s \models \sigma\} \Rightarrow\left\{S_{j}\right\}
$$


The decision on how situations should be defined and how the application space should be initialized are application-specific. The framework presented in Section 3 thus allows corresponding configurations.

\section{Incompleteness and Ambiguity in the Situation Model}

The previously presented inference function of CST is based on the evidential Dempster-Shafer approach [18] to calculate confidence levels, which has also been used in ECSTRA [4]. The inference function in CST is defined as a placeholder function that can be substituted by other approaches which can apply other well-known reasoning techniques, as for example shown with the Bayesian approach [16]. Techniques applied for reasoning have different strengths in covering uncertain aspects like incompleteness or ambiguity. The application of both, evidence theory and fuzzy logic, for example is capable of handling incomplete, imprecise and out of date contextual information [23]. Thus, the design requirements for knowledge base is dependent on the input that the inference function requires. Based on the inference function presented in Section 4, the ontology requires extension regarding the confidence thresholds (specified for situations), relevance functions (specified for attributes per situation definition), and contribution functions (specified for attribute values), as presented in [14]. The incorporation of the relevance function into the infon definition, for example, can be formally denoted as $\sigma=\ll r,\left(a_{1}, w_{1}\right), \ldots,\left(a_{n}, w_{n}\right), i \gg$, where $w_{n}$ represents the weight for the attribute $a_{n}$.

The availability of knowledge is a major concern for specification-based approaches. Domain experts are required to describe the relevant situations beforehand. The contribution function and the relevance function in the presented approach already allow modelling of situations with imprecise knowledge. With STO as an underlying situation model, this aspect can be even further improved. The integration of the earlier introduced related approach of Fuzzy STO [11], for example, proposes a non-numerical specification in case of vague knowledge about a situation. In FSTO, the polarity $i$ of infons is replaced with $\tau$ which is element of a grammar that allows statements like quite, less, really true or false.

Even though both approaches, adding the weight $w_{n}$ assigned to attributes from CST and extending the polarity with $\tau$ in FSTO, extend the infon definition to handle uncertain aspects due to incompleteness of knowledge, they are semantically not equal. Whereas the weight assigns a relative importance to the attributes, $\tau$ makes a statement about the truth of the overall infon, which could affect a combination of objects. Integrating both approaches would increase the imprecise modelling capabilities for domain experts. The corresponding extended inference function for CST is shown in Eq. (4) in which $\tau_{\sigma}\left(a_{i}\right)$ represents the confidence in the infon truth of the supported infon in which the attribute $a_{i}$ is involved.

$$
\mu_{S_{j}}=\sum_{i=1}^{n} w_{S_{j}}\left(a_{i}\right) * \eta_{a_{i}}^{S}\left(x_{a_{i}}\right) * \tau_{\sigma}\left(a_{i}\right)
$$


Another issue of situation aware systems is the absence of knowledge during the modelling process. This is a major concern for any specification- or learning-based approach, either in the form of missing situation definitions or incompleteness in the training data. It is a difficult challenge to react properly to an unknown situation, i.e. when the contextual circumstances cannot be interpreted with the provided knowledge. In CST, this occurs when the context state reaches a point in the context space which is not in any of the known situation spaces. The following measures can be considered to address these issues.

Verifying completeness. The knowledge base could be analyzed before the deployment of the system to identify gaps. For the presented framework, this could be done by looking for gaps in acceptable regions for context attributes.

Reasoning-supported completion. Previous and following situations, situations with same context attributes, and situations close to the context state can give a semantic indication to the domain expert when a gap in the context space is encountered during run-time.

Accessing remote repositories. The possibility to look up situation definitions stored in remote, standardized repositories, e.g. with semantic web technologies, might allow to automatically close the gaps for undefined situations.

To summarize, handling uncertainty in the knowledge base of a situation aware system is a necessary step to enable reliable reasoning and to provide a practical approach to represent domain knowledge. Identifying and resolving gaps in the knowledge base is important to avoid malfunctions of the overall situation aware system.

\section{$6 \quad$ IoT Interoperability and Context Adaptation}

Situation aware systems rely on the access to contextual information of the environment. IoT interoperability aims to integrate data from various sources (like sensor networks, files, APIs and so on) across different platforms. Thus, the integration of relevant standards in the overall situation aware system is investigated. Furthermore, the dynamic aspect of the environment has also an impact on the situation model. In particular, adapting to current context will be discussed.

\subsection{IoT Interoperability with O-MI/O-DF Integration}

Interoperability across heterogeneous platforms is a major concern for the IoT. Vertical silos prevent communication between different platforms on several levels, limiting the access to contextual information [9]. As previously mentioned, several H2020 projects (as part of the IoT-European Platforms Initiative) are working on this challenge by developing protocols, tools, and platforms that provide communication interfaces on top of well-established IoT communication protocols 
(such as MQTT, CoAP, HTTP, etc.). The framework presented in this paper relies on the open standards O-MI and O-DF, which are developed in the H2020 bIoTope project. O-MI provides a messaging interface that can be used on top of session protocols, which includes operations like read, write and subscribe. O-DF, on the other hand, provides a generic service description model for IoT services, in which the message payload is structured as a tree of objects and their infoitems with corresponding values and metadata.

The integration of this standardized API and the upper ontology allows for automation of processes and thus reduces the implementation effort for context collection of the reasoning engine. Furthermore, the final situation awareness framework can be applied independent of the platforms and technologies that are used on site. Solely configuration files are required to map the O-DF data stream to objects that are defined in the ontology (sensors, attributes, etc.). The situation spaces and the context collectors are both automatically generated upon initialization of the system. Context collectors start to subscribe (event-based) to the sensor data streams using the O-MI subscription mechanisms. During run-time, the O-DF payload is resolved with the semantic model of the ontology, which allows to update the context state without the development of custom modules. This enables automated reasoning with minimal implementation effort for client applications, considering a standardized IoT embedment.

\subsection{Adaptation of the Knowledge Base to Current Context in a Dynamic Environment}

Situation aware systems are often deployed in dynamic environments, which requires the knowledge base to adapt to current context. The following two dynamic aspects that have a direct impact on the knowledge base are considered, (i) involved individuals entering or leaving the system's scope, and (ii) sensors entering or leaving the system's scope

Adapting to these changes does not only imply maintaining the knowledge base (e.g. adding and removing involved individuals and sensors) but also maintaining the application space (e.g. adding and removing situation spaces). Since the framework already provides situation awareness, the adaptation mechanism can be i ntegrated on top of the existing functionalities in a straight-forward manner. Individuals or sensors that are entering or leaving the system can be perceived as a contextual information that is provided by sensors.

Recognizing and clearly identifying involved individuals in situations might require additional sensing, e.g. sensed smartphone locations and gathered user profiles could be used to track the presence of persons (which can be also exposed and described through O-MI/O-DF gateways). The different events of tracking individuals and sensors can be modelled as situations. Upon the occurrence of such a situation the framework automatically takes all necessary steps (regarding knowledge base and application space) to adapt the system to the new circumstances. 


\section{Conclusion and Future Work}

This paper discussed the incorporation of an ontology as a knowledge base for a situation aware framework. Detailed explanation of the mapping of Situation Theory and CST demonstrated the enrichment of a reasoning framework with a situation model based on STO. The discussions related to a knowledge base for situation aware systems further included the required knowledge assets that must be provided to allow reasoning, modelling incomplete knowledge, handling unknown situations, cross-domain and -platform interoperability issues in the IoT, and adaptation to current context.

In conclusion, the situation model could be extended to accommodate all relevant assets and to enhance the run-time reasoning. Moreover, the knowledge base allows modelling with imprecise knowledge and allows the tracking of semantic changes of the system's environment and to provide situation awareness in a dynamic environment.

Future work includes the development of use cases to compare different approaches to uncertainty modelling and resolving unknown situations. Furthermore, the framework could be enhanced by integrating learning-based techniques. The challenge of meeting run-time reasoning requirements, performance and scalability expectations is as well an important part of future work.

\section{Acknowledgment}

Part of this work has been carried out in the scope of the project bIoTope which is co-funded by the European Commission under Horizon-2020 program, contract number H2020-ICT-2015/ 688203 - bIoTope. The research has been carried out with the financial support of the Ministry of Education and Science of the Russian Federation under grant agreement RFMEFI58716X0031.

\section{References}

1. Abowd, G.D., Dey, A.K., Brown, P.J., Davies, N., Smith, M., Steggles, P.: Towards a better understanding of context and context-awareness. In: International Symposium on Handheld and Ubiquitous Computing. pp. 304-307. Springer (1999)

2. Baumgartner, N., Retschitzegger, W.: A survey of upper ontologies for situation awareness. In: Proc. of the 4th IASTED International Conference on Knowledge Sharing and Collaborative Engineering, St. Thomas, US VI. pp. 1-9 (2006)

3. Bettini, C., Brdiczka, O., Henricksen, K., Indulska, J., Nicklas, D., Ranganathan, A., Riboni, D.: A survey of context modelling and reasoning techniques. Pervasive and Mobile Computing 6(2), 161-180 (2010)

4. Boytsov, A., Zaslavsky, A.: Ecstra-distributed context reasoning framework for pervasive computing systems. In: Smart Spaces and Next Generation Wired/Wireless Networking, pp. 1-13. Springer (2011)

5. Boytsov, A., Zaslavsky, A., Eryilmaz, E., Albayrak, S.: Situation Awareness Meets Ontologies: A Context Spaces Case Study, pp. 3-17. Modeling and Using Context, Springer (2015) 
6. Boytsov, A., Zaslavsky, A., Synnes, K.: Extending context spaces theory by predicting run-time context. In: Smart spaces and next generation wired/wireless networking, pp. 8-21. Springer (2009)

7. Chen, H., Finin, T., Joshi, A.: The SOUPA ontology for pervasive computing, pp. 233-258. Ontologies for agents: Theory and experiences, Springer (2005)

8. Compton, M., Barnaghi, P., Bermudez, L., García-Castro, R., Corcho, O., Cox, S., Graybeal, J., Hauswirth, M., Henson, C., Herzog, A., et al.: The ssn ontology of the w3c semantic sensor network incubator group. Web semantics: science, services and agents on the World Wide Web 17, 25-32 (2012)

9. Desai, P., Sheth, A., Anantharam, P.: Semantic gateway as a service architecture for iot interoperability. In: 2015 IEEE International Conference on Mobile Services. pp. 313-319. IEEE (2015)

10. Devlin, K.: Situation theory and situation semantics. Handbook of the History of Logic 7, 601-664 (2006)

11. Furno, D., Loia, V., Veniero, M.: A fuzzy cognitive situation awareness for airport security. Control and cybernetics 39(4), 959-982 (2010)

12. Gruber, T.R.: Toward principles for the design of ontologies used for knowledge sharing? International journal of human-computer studies 43(5), 907-928 (1995)

13. Kokar, M.M., Matheus, C.J., Baclawski, K.: Ontology-based situation awareness. Information fusion 10(1), 83-98 (2009)

14. Kolbe, N., Zaslavsky, A., Kubler, S., Robert, J.: Reasoning over knowledge-based generation of situations in context spaces to reduce food waste. In: International Conference on Next Generation Wired/Wireless Networking. pp. 101-114. Springer (2016)

15. Matheus, C.J., Kokar, M.M., Baclawski, K., Letkowski, J.A., Call, C., Hinman, M.L., Salerno, J.J., Boulware, D.M.: Sawa: An assistant for higher-level fusion and situation awareness. In: Defense and Security. pp. 75-85. International Society for Optics and Photonics (2005)

16. Padovitz, A.: Context management and reasoning about situations in pervasive computing. Monash University Melbourne (2006)

17. Padovitz, A., Loke, S.W., Zaslavsky, A.: Towards a theory of context spaces. In: Pervasive Computing and Communications Workshops, 2004. Proceedings of the Second IEEE Annual Conference on. pp. 38-42. IEEE (2004)

18. Shafer, G.: A mathematical theory of evidence, vol. 1. Princeton University Press, Princeton (1976)

19. Spalazzi, L., Taccari, G., Bernardini, A.: An internet of things ontology for earthquake emergency evaluation and response. In: Collaboration technologies and systems (CTS), 2014 International conference on. pp. 528-534. IEEE (2014)

20. Strang, T., Linnhoff-Popien, C.: A context modeling survey. In: Workshop on Advanced Context Modelling, Reasoning and Management, UbiComp 2004 (2004)

21. Wang, X.H., Zhang, D.Q., Gu, T., Pung, H.K.: Ontology based context modeling and reasoning using owl. In: Pervasive Computing and Communications Workshops, 2004. Proceedings of the Second IEEE Annual Conference on. pp. 18-22. IEEE (2004)

22. Yau, S.S., Liu, J.: Hierarchical situation modeling and reasoning for pervasive computing. In: Software Technologies for Future Embedded and Ubiquitous Systems, 2006 and the 2006 Second International Workshop on Collaborative Computing, Integration, and Assurance. SEUS 2006/WCCIA 2006. The Fourth IEEE Workshop on. p. 6 pp. IEEE (2006)

23. Ye, J., Dobson, S., McKeever, S.: Situation identification techniques in pervasive computing: A review. Pervasive and Mobile Computing 8(1), 36-66 (2012) 